\title{
BMJ Open Impact of multimorbidity count on all- cause mortality and glycaemic outcomes in people with type 2 diabetes: a systematic review protocol
}

\author{
Jason I Chiang, ${ }^{1}$ John Furler, ${ }^{1}$ Frances S Mair, ${ }^{2}$ Bhautesh Jani, ${ }^{2}$ Barbara I Nicholl, ${ }^{2}$ \\ Alicia Jenkins, ${ }^{3}$ Patrick Condron, ${ }^{4}$ David O'Neal, ${ }^{5}$ Jo-Anne Manski-Nankervis ${ }^{1}$
}

To cite: Chiang Jl, Furler J, Mair FS, et al. Impact of multimorbidity count on allcause mortality and glycaemic outcomes in people with type 2 diabetes: a systematic review protocol. BMJ Open 2018;8:e21100. doi:10.1136/ bmjopen-2017-021100

- Prepublication history and additional material for this paper are available online. To view these files, please visit the journal online (http://dx.doi. org/10.1136/bmjopen-2017021100).

Received 11 December 2017 Revised 15 March 2018 Accepted 20 March 2018
Check for updates

${ }^{1}$ Department of General Practice, University of Melbourne, Melbourne, Victoria, Australia ${ }^{2}$ General Practice and Primary Care, Institute of Health and Wellbeing, University of Glasgow, Glasgow, UK ${ }^{3}$ NHMRC Clinical Trials Centre, University of Sydney, Sydney, New South Wales, Australia ${ }^{4}$ Brownless Biomedical Library, University of Melbourne, Melbourne, Victoria, Australia ${ }^{5}$ Department of Medicine, St Vincent's Hospital, University of Melbourne, Melbourne, Victoria, Australia

Correspondence to

Jason I Chiang;

jason.chiang@unimelb.edu.au

\section{ABSTRACT}

Introduction Type 2 diabetes (T2D) is a leading health priority worldwide. Multimorbidity (MM) is a term describing the co-occurrence of two or more chronic diseases or conditions. The majority of people living with T2D have MM. The relationship between MM and mortality and glycaemia in people with T2D is not clear.

Methods and analysis Medline, Embase, Cumulative Index of Nursing and Allied Health Complete, The Cochrane Library, and SCOPUS will be searched with a prespecified search strategy. The searches will be limited to quantitative empirical studies in English with no restriction on publication date. One reviewer will perform title screening and two review authors will independently screen the abstract and full texts using Covidence software, with disagreements adjudicated by a third reviewer. Data will be extracted using a using a Population, Exposure, Comparator and Outcomes framework. Two reviewers will independently extract data and undertake the risk of bias (quality) assessment. Disagreements will be resolved by consensus. A narrative synthesis of the results will be conducted and meta-analysis considered if appropriate. Quality appraisal will be undertaken using the Newcastle-Ottawa quality assessment scale and the quality of the cumulative evidence of the included studies will be assessed using the Grading of Recommendations, Assessment, Development and Evaluation approach. This protocol was prepared in adherence to the Preferred Reporting Items for Systematic Reviews and Meta-Analyses Protocols guidelines to ensure the quality of our review. Ethics and dissemination This review will synthesise the existing evidence about the impact of MM on mortality and glycaemic outcomes in people living with T2D and increase our understanding of this subject and will inform future practice and policy. Findings will be disseminated via conference presentations, social media and peerreviewed publication.

PROSPERO registration number CRD42017079500.

\section{INTRODUCTION}

\section{Rationale}

Type 2 diabetes (T2D) is a major health priority of the 21 st century. Worldwide, it is estimated that more than 424 million people live with diabetes, resulting in
Strengths and limitations of this study

- This will be the first systematic review to explore the impact of multimorbidity (MM) on all-cause mortality and glycaemia in people with type 2 diabetes (T2D) and has the potential to make a valuable contribution to the literature in this area.

- Our review benefits from a comprehensive search strategy including key terms, synonyms and medical subject headings that describe the research questions with a deliberate inclusion of the 'comorbidity' term to address the identified issue of the terms 'comorbidity' and 'multimorbidity' being used interchangeably.

- We expect the literature to be quite heterogeneous in terms of how MM is defined and the way outcomes are reported so that a narrative synthesis may be likely.

US $\$ 727$ billion in healthcare expenditures. ${ }^{1}$ Approximately 4 million people die from diabetes-related causes each year, equivalent to 1 death every $8 \mathrm{~s}$, with nearly half of these deaths in people under the age of $60 .^{1}$ There is no doubt that T2D imposes a heavy burden on communities.

The management of T2D is complex, requiring continuous efforts to implement recommendations for self-management and pharmacotherapy in a stepwise manner to achieve evidence-based targets. This complexity is increased when the patient has other chronic conditions in addition to T2D because T2D rarely occurs on its own. Data suggest that as many as $85 \%$ of those with T2D have at least one other chronic condition, ${ }^{2}$ which is higher than the $52 \%$ in the general population that is multimorbid. ${ }^{3}$

For many years, the terms comorbidity and multimorbidity (MM) were used interchangeably. ${ }^{4}$ It has only been more recently that there has been a clearer distinction and understanding between the two terms. 
Comorbidity is defined as the existence or occurrence of any additional condition(s) that co-occurs with an index disease. ${ }^{5} \mathrm{MM}$ however refers to the presence of two or more chronic conditions in an individual, with no reference to an index condition. ${ }^{6}$ These established definitions provide the basis of our systematic review which exclusively focuses on MM in T2D.

MM presents multiple challenges. It is associated with a reduced quality of life, increased costs, a reduced ability to make lifestyle changes and may be associated with complex therapeutic regimens which the patient may be challenged to manage. ${ }^{7}$ For health professionals, MM brings increased workload and the clinical challenges of interactions between multiple conditions and medications. ${ }^{4}$ Most condition-specific management guidelines do not account for MM and prioritise management of one condition at the expense of another. ${ }^{7}$ For people with diabetes, this can lead to clinicians focusing on diabetes only without consideration of the patient's other conditions and patient goals. Similarly, a focus on other conditions may lead to suboptimal glycaemic management due to a lack of focus on diabetes-specific care goals. ${ }^{8}{ }^{9}$ This is particularly problematic because achieving and maintaining glycaemic targets early is important in reducing downstream complications and all-cause mortality. ${ }^{10}$

Currently, little is known about the associations between MM and T2D. In particular, there is little information regarding the relationship of the total burden of disease reflected in MM's multiple dimensions to the association between all-cause mortality and glycaemia.

Our systematic review will focus on current knowledge regarding the impact of $\mathrm{MM}$ on mortality and glycaemia in people with T2D and provide insights regarding the implications of MM in the context of this chronic disease. It may provide an important foundation of knowledge for improving care for patients with T2D and multiple chronic conditions.

\section{Objectives}

The primary objective of our systematic review is to determine the impact of MM reflected in condition count on all-cause mortality and glycaemia in people with T2D. We will have two primary outcomes of equal interest: (1) all-cause mortality; and (2) glycaemia (measured by glycated haemoglobin (HbA1c)).

Secondary outcomes of interest include: (1) hypoglycaemia, (2) hyperglycaemia and (3) glycaemic variability.

\section{METHODS AND ANALYSIS}

The Preferred Reporting Items for Systematic Reviews and Meta-Analyses Protocols (PRISMA-P) guidelines has been used to prepare this protocol. ${ }^{11}$

\section{Eligibility criteria}

\section{Study characteristics/design}

All quantitative empirical studies published in the English language will be included. Our target studies will be observational studies that use either longitudinal cohort (retrospective and prospective) or cross-sectional designs. While we recognise the limitation of cross-sectional studies in terms of assessment of temporality, cross-sectional studies provide a snapshot of the association between MM count and our glycaemia-related outcomes of interest. We will have no restrictions on publication date. The search end date will be 28 July 2017.

Randomised controlled trials, non-diabetes drug intervention studies, all qualitative studies, case reports, review articles and conference abstracts will be excluded as they will not give us information on our primary outcomes of interest. Randomised controlled trials and non-diabetes drug intervention studies have primary objectives of testing particular interventions so the effect of MM will not be captured, thus inappropriate for our review which is focused on the effects on MM. All non-English studies will also be excluded.

\section{Population}

Our target study population is adults (18 years of age or older) with T2D.

Studies including populations of children and adolescents (under 18 years of age) or people without T2D (eg, people with prediabetes, type 1 diabetes/gestational diabetes/monogenic diabetes) will be excluded. Animal studies will also be excluded.

\section{Exposure}

The primary exposure of interest is MM count. We will accept any type of MM count, which may include a list of chronic conditions from a variety of datasets including electronic medical records, administrative and prescription datasets. Only studies that assess the relationship

Table 1 Search terms

\begin{tabular}{|c|c|c|c|}
\hline Key terms & Multimorbidity & Diabetes & $\begin{array}{l}\text { Outcomes of } \\
\text { interest: } \\
\text { mortality } \\
\text { glycaemia }\end{array}$ \\
\hline $\begin{array}{l}\text { Other } \\
\text { related } \\
\text { terms or } \\
\text { synonyms }\end{array}$ & 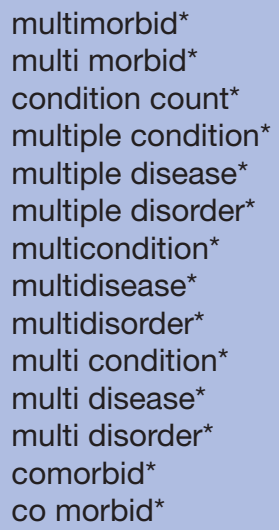 & $\begin{array}{l}\text { diabet }^{*} \\
\text { diabetes } \\
\text { adj2 } \\
\text { (type } 2 \text { or } \\
\text { type ii) }\end{array}$ & $\begin{array}{l}\text { mortality } \\
\text { death } \\
\text { surviv* }^{*} \\
\text { surviv*}^{*} \\
\text { analys* } \\
\text { glycaemia* } \\
\text { glycemia* } \\
\text { hypoglycaem* } \\
\text { hypoglycem* } \\
\text { hyperglycaem* } \\
\text { hyperglycem* } \\
\text { glycem* }{ }^{*} \text { varia* }^{*} \\
\text { glycaem* } \text { varia* }^{*}\end{array}$ \\
\hline
\end{tabular}


between a numerical count of MM and our outcomes of interest will be included.

Studies with single nominated specific conditions (ie, only one comorbid condition) linked with T2D without MM count will be excluded.

\section{Comparators (control)}

A comparator/control group is defined by people with T2D with no other chronic conditions. Studies that do not include such a control group will not be excluded.

\section{Outcome}

A study will be included in our review if data is provided regarding either all-cause mortality or glycaemic outcomes. It is expected that glycaemia will be reported in the form of $\mathrm{HbAlc}$, however, we will include any measure of glycaemia, for example, hypoglycaemia, hyperglycaemia or glycaemic variability.

\section{Information sources}

We will search five electronic databases including Medline (Ovid interface), Embase (Ovid interface), Cumulative Index of Nursing and Allied Health Complete (EBSCO interface), The Cochrane Library (Ovid interface) and SCOPUS with no restrictions on publication date.

We will check the International Prospective Register of Systematic Reviews regularly for ongoing and completed systematic reviews for MM and T2D.

\section{Search strategy}

The search strategy will include medical subject headings, terms and synonyms relating to or describing our primary objectives. These terms will be combined using appropriate Boolean logic operators to create our search strategy. The truncation symbol (*) will also be included at the end of the stem of a word to retrieve all words that start with that stem. Our strategy has been reviewed by a librarian from a biomedical library and members of our review panel with expertise in MM and T2D. A number of test runs will first be conducted with Medline, and any necessary adjustments will be made prior to running the search. Once the search strategy is finalised, the searches will be adapted for each of the five electronic databases prior to conducting the searches. The search terms are listed in table 1 . The full search strategy is available in online supplementary file 1 .

\section{Study records}

Data management

Literature search results will be downloaded to EndNote (V.7; Clarivate Analytics) and duplicates will be removed. The non-duplicate studies will then be uploaded to Covidence, ${ }^{12}$ a systematic review management software, for the selection process.

\section{Selection process}

The selection process of the studies for inclusion in our review will be conducted in three stages. First, titles of the studies identified in the five database searches will be screened by the primary researcher (JC) against the predefined eligibility criteria outlined above. A deliberately inclusive approach will be adopted for this title screening stage to reduce the risk of missing potentially relevant studies.

Second, all abstracts will be screened by two reviewers independently. The primary researcher (JC) will screen all abstracts against the predefined eligibility criteria outlined above to identify a subset of potentially relevant studies. An independent second screening of the abstracts will be completed between the following reviewers (JMN, JF, BN, BJ, AJ, FM). Any inter-reviewer disagreements will be discussed and resolved by a third reviewer (JMN, JF).

Finally, we will obtain full-text articles for all studies that appear to meet our eligibility criteria after the title and abstract screening stages. Full-text screening will be conducted by two reviewers independently. The primary researcher (JC) will screen all full texts against our predefined eligibility criteria. This will then be repeated independently by a second reviewer (JMN, JF, BJ). Online supplementary material will be consulted when necessary. Again, any inter-reviewer disagreements will be discussed and resolved by a third reviewer (JMN, JF). Reasons for exclusion at the full-text screening process will be recorded.

\section{Data extraction}

Data will be extracted in a structured manner from all included studies and recorded in a predefined data extraction form designed by the primary researcher (JC) following a prespecified Population, Exposure, Comparator, Outcomes framework in the data extraction stage. This is an adapted framework based on the Cochrane PICO statement where 'I' for intervention is replaced with an ' $\mathrm{E}$ ' for exposure. We will also be including an extra 'study characteristics' parameter to record characteristics of the study including study design, setting, period of study and aims and objectives (see online supplementary file 2). The form will be reviewed, refined and adjusted where necessary by the review team. Again, online supplementary material will be consulted when necessary for data extraction.

\section{Data items}

We will be extracting relevant data in each of the following five parameters:

\section{Populations}

We will extract data on characteristics of study populations (sample size, sex, age, ethnicity, socioeconomic status, occupation, education, diabetes duration, HbA1c, insulin treatment and oral antidiabetes drugs), as well as definition/measure of T2D, method of recruitment and sampling and inclusion and exclusion criteria.

\section{Exposure}

We will describe the definition/measure of MM count and number of subjects reported. 


\section{Comparator}

We will provide details provided in the publication of any comparator groups including the definition/measure of people with T2D with no other chronic/long-term conditions and numbers in group.

\section{Outcomes}

We will provide details as to how all-cause mortality and/ or glycaemia is defined/measured, as well as length of follow-up, number of subjects and the statistical analyses employed by the authors to evaluate the relationship between MM count and the measured outcomes.

\section{Study characteristics}

We will extract details of study design, setting, period of study and aims and objectives.

\section{Outcomes and prioritisation}

One of the primary clinical outcomes of interest is all-cause mortality. We expect that studies will calculate the effect estimate as either HRs, ORs, incidence rates or survival percentages.

For our other primary outcome, glycaemia, we will prioritise those studies that measure glycaemia in terms of HbA1c. We will further divide studies into one of two groups: those that measure $\mathrm{HbAlc}$ as a continuous variable and those that measure $\mathrm{HbAlc}$ as a categorical variable.

We will accept all other measures of glycaemia as secondary outcomes.

\section{Risk of bias assessment (quality assessment in individual studies)}

Two reviewers will independently assess the risk of bias (quality) in each of the included studies.

All studies will be assessed using the Newcastle-Ottawa quality assessment scale. ${ }^{13}$ The choice of this tool was informed by recommendations from the Cochrane Handbook on assessing the quality of non-randomised studies. ${ }^{14}$ Any inter-reviewer disagreements will be discussed and resolved by a third reviewer.

The Newcastle-Ottawa quality assessment scale has a star system to judge three broad perspectives of the included studies: the selection of the study groups; the comparability of the groups and the ascertainment of the outcome of interest.

\section{Data synthesis}

For data synthesis, we will group the included studies according to the two outcomes of all-cause mortality and glycaemia. Within the glycaemia outcome group, we will further subgroup the studies into the different measures of glycaemia. For our primary analysis, we will consider either all-cause mortality or glycaemia each as a composite outcome. However, dependent on the number of studies retrieved, an analysis of glycaemia subtype will be conducted. Furthermore, dependent on the characteristics of the study populations, we will consider stratifying our results according to either exposure ascertainment
(MM count) or population characteristics (ie, age group, gender and socioeconomic status).

A narrative synthesis of findings will be conducted which will describe the findings from each of the included studies. For each study we will present details relating to the following:

- The number and characteristics of participants in the study.

- Setting.

- Study design.

- The outcome-level risk of bias of the study.

- Findings for quantitative outcomes.

- Inconsistent findings within individual studies will be indicated.

If further information relevant to our review is required, we will attempt to contact the authors of the included studies.

A meta-analysis will be conducted if appropriate. Tests for publication bias and heterogeneity will be conducted. If the included studies are sufficiently homogenous in terms of study design, study population, outcomes and data analysis, a meta-analysis will be considered. $\mathrm{I}^{2}$ statistic will be used to assess statistical heterogeneity and to guide the choice of either fixed or random effects model. Given sufficient numbers of included studies, a funnel plot will be used to assess publication bias and other reporting bias, and a Begg's test will be used to test for asymmetry. A sensitivity analysis will also be used to determine the consistency of the results. However, if a meta-analysis and the above tests are not possible, possible sources of bias across studies will be discussed in the narrative synthesis, and this limitation will be considered when drawing conclusions.

\section{Confidence in cumulative evidence}

Grading of Recommendations, Assessment, Development and Evaluation (GRADE) guideline is recommended by the Cochrane Handbook for grading the quality and strength of evidence. ${ }^{15}$ We will use the GRADE guidelines to assess the quality of evidence for our research questions.

\section{Patient and public involvement}

Patients were not involved in the development of this protocol.

\section{ETHICS AND DISSEMINATION \\ Ethics}

Ethics was not required for this study as this is a systematic review and it does not contain individual patient data. We will disseminate the results of our review via conference presentations, social media and peer-reviewed publication. This review also forms part of the lead investigator's (JC) PhD.

\section{DISCUSSION}

This systematic review will aim to synthesise the existing evidence on the effects of MM in T2D on mortality and 
glycaemic control and will be the first on this subject. Clinical management in patients with T2D and MM is a growing international healthcare challenge, and our review will make important contributions to understanding of the impact of MM, if any, in the context of T2D on key clinical outcomes which should enhance the understanding in this field. We hypothesise that increasing MM will be associated with increased all-cause mortality; however, the effects on glycaemic outcomes may vary. Our review will be the first to bring together existing literature exploring associations between MM and T2D, and therefore clarifying the effects of increasing MM on mortality and glycaemia in people with T2D.

Key strengths of our review will be our adherence to the PRISMA-P guidelines, our comprehensive search strategy and the fact that all screening and data extraction will be performed by two reviewers independently. We expect the literature to be quite heterogeneous in terms of how MM is defined and the way outcomes are reported so a narrative synthesis may be likely which may be a limitation. In addition, we have restricted our review to English language publications which is a potential limitation.

As the first review on this subject, it will help identify what is known on this subject and whether any gaps in knowledge exist. It will therefore help highlight whether there are areas requiring further investigation as well as clarify the key messages from the evidence, to date, including implications for future guidelines for those with T2D.

Contributors JC drafted the protocol and developed the search strategy, inclusion/ exclusion criteria and the data extraction form with guidance from JMN, JF, FM, BN, BJ, AJ and DO. PC contributed to the development of the search. All coauthors read and provided feedback on the draft manuscript. All authors approved the manuscript for submission.

Funding This research received no specific grant from any funding agency in the public, commercial or not-for-profit sectors.

Competing interests None declared.

Patient consent Not required.

Provenance and peer review Not commissioned; externally peer reviewed.
Open Access This is an Open Access article distributed in accordance with the Creative Commons Attribution Non Commercial (CC BY-NC 4.0) license, which permits others to distribute, remix, adapt, build upon this work non-commercially, and license their derivative works on different terms, provided the original work is properly cited and the use is non-commercial. See: http://creativecommons.org/ licenses/by-nc/4.0/

(c) Article author(s) (or their employer(s) unless otherwise stated in the text of the article) 2018. All rights reserved. No commercial use is permitted unless otherwise expressly granted.

\section{REFERENCES}

1. International Diabetes Federation. IDF diabetes atlas. 8th edn, 2017.

2. Australian Bureau of Statistics. National Health Survey: First Result 2015;15.

3. Harrison C, Henderson J, Miller G, et al. The prevalence of diagnosed chronic conditions and multimorbidity in Australia: a method for estimating population prevalence from general practice patient encounter data. PLoS One 2017;12:e0172935.

4. Fortin M, Lapointe L, Hudon C, et al. Multimorbidity is common to family practice: is it commonly researched? Can Fam Physician 2005;51:244-5.

5. Feinstein AR. The pre-therapeutic classification of co-morbidity in chronic disease. J Chronic Dis 1970;23:455-68.

6. Barnett K, Mercer SW, Norbury M, et al. Epidemiology of multimorbidity and implications for health care, research, and medical education: a cross-sectional study. Lancet 2012;380:37-43.

7. Harris MF, Dennis S, Pillay M. Multimorbidity: negotiating priorities and making progress. Aust Fam Physician 2013;42:850-4.

8. Piette JD, Kerr EA. The impact of comorbid chronic conditions on diabetes care. Diabetes Care 2006;29:725-31.

9. Boyd CM, Fortin M. Future of multimorbidity research: how should understanding of multimorbidity inform health system design? Public Health Rev 2011;33:451-74.

10. Holman RR, Paul SK, Bethel MA, et al. 10-year follow-up of intensive glucose control in type 2 diabetes. N Engl J Med 2008;359:1577-89.

11. Moher D, Shamseer L, Clarke M, et al. Preferred Reporting Items for Systematic Review and Meta-Analysis Protocols (PRISMA-P) 2015 statement. Syst Rev 2015;4:1.

12. Veritas Health Innovation, Melbourne, Australia. Covidence systematic review software. www.covidence.org.

13. Wells G, Shea B, O'Connell D, et al. The Newcastle-Ottawa Scale (NOS) for assessing the quality of nonrandomised studies in metaanalyses. http://www.ohri.ca/programs/clinical_epidemiology/oxford. asp (cited $28 \mathrm{Jul} 2017$ ).

14. Higgins J, Green S. Cochrane Handbook for Systematic Reviews of Interventions version 5.1.0 [updated March 2011]. 2011 http:// handbook.cochrane.org

15. GRADE. Grading of Recommendations Assessment, Development and Evaluation (GRADE) Working Group GRADE Working Group. http://www.gradeworkinggroup.org/ (cited 3 Aug 2017). 